\title{
Recognition of shorter and longer trimethyllysine analogues by epigenetic reader proteins
}

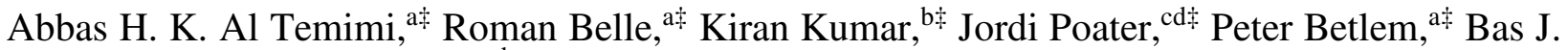
G. E. Pieters, ${ }^{\mathrm{a}}$ Robert S. Paton, ${ }^{\mathrm{b}}$ F. Matthias Bickelhaupt ${ }^{\mathrm{ae}}$ and Jasmin Mecinović*a

${ }^{a}$ Institute for Molecules and Materials, Radboud University, Heyendaalseweg 135, Nijmegen 6525 AJ, The Netherlands. E-mail: j.mecinovic@ science.ru.nl; Fax: +31 24 3653393; Tel: +31 24 3652381

${ }^{\mathrm{b}}$ Chemistry Research Laboratory, University of Oxford, 12 Mansfield Road, Oxford OX1 3TA, UK

c ICREA, Pg. Llu1's Companys 23, Barcelona 08010, Spain

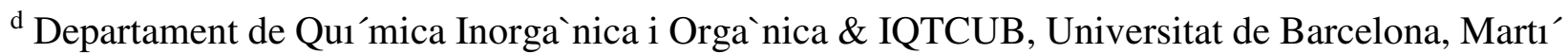
i Franque`s 1-11, Barcelona 08028, Spain

${ }^{\mathrm{e}}$ Department of Theoretical Chemistry and Amsterdam Center for Multiscale Modeling (ACMM), Vrije Universiteit Amsterdam, De Boelelaan 1083, Amsterdam 1081 HV, The Netherlands

$\dagger$ Electronic supplementary information (ESI) available: Supporting figures, synthesis, ITC data, MDS data, and computational data.

\$ These authors contributed equally to this work.

Histone $N^{\varepsilon}$-lysine methylation is a widespread posttranslational modification that is specifically recognised by a diverse class of $N^{\varepsilon}$-methyllysine binding reader proteins. Combined thermodynamic, molecular dynamics simulations, and quantum chemical studies reveal that reader proteins efficiently bind trimethylornithine and trimethylhomolysine, the simplest $N^{\varepsilon}$-trimethyllysine analogues that differ in the length of the side chain.

Methylation of lysine residues in histone proteins plays an important role in the regulation of genes in humans and other eukaryotes. ${ }^{1,2}$ Histone $N^{\varepsilon}$-lysine methylation is catalysed by a large number of S-adenosylmethionine dependent histone lysine methyltransferases (KMTs), and can lead to the formation of monomethylated (KMe), dimethylated (KMe2) or trimethylated (KMe3) lysine residues. ${ }^{3}$ The opposite reaction, i.e. the removal of the $N^{\varepsilon}$-methyl group(s) from methylated lysines, is catalysed by either flavin dependent or non-haem Fe(II) and 2-oxoglutarate dependent histone lysine demethylases (KDMs). ${ }^{4}$ Each methylation mark is specifically recognised by structurally diverse family of $N^{\varepsilon}$-methyllysine binding proteins (also known as reader proteins), including plant homeodomain (PHD) zinc fingers (Fig. 1A), tandem tudor domains (TTD, Fig. 1B) and chromodomains. ${ }^{5}$ The association between the positively charged KMe3 and the electronrich aromatic cage-containing reader proteins is primarily driven by a combination of energetically 
favourable cation $-\pi$ interactions ${ }^{6,7}$ and the trimethyllysine-mediated release of high-energy water molecules that occupy the aromatic cages. ${ }^{7}$

Epigenetic readers of trimethyllysine exhibit selectivity for the highest methylation mark over lower methylation states; in general, the trend of binding affinities is $\mathrm{KMe} 3>\mathrm{KMe} 2>\mathrm{KMe}$ $>$ K. ${ }^{5,8}$ Comparative binding analyses of histone peptides that contain L-KMe3 and D-KMe3 confirmed the preference of reader proteins for the association with lysine that possesses the Lstereochemistry. ${ }^{9}$ Members of reader proteins also associate with the analogous cysteine-derived trimethyllysine in histone peptides and intact histone proteins. ${ }^{10,11}$ Recent thermodynamic, structural, and computational work also revealed that the neutral carba analogue of trimethyllysine is recognised by several members of reader proteins. ${ }^{7}$ At present, however, the effect of the lysine chain length on the epigenetic readout is lacking. Herein, we report comparative thermodynamic analyses, molecular dynamics simulations, and quantum chemical analyses on the epigenetic readout of $N^{\varepsilon}$-trimethyllysine (KMe3) and its two simplest analogues, the shorter trimethylornithine (OrnMe3) and the longer trimethylhomolysine (hKMe3) (Fig. 1C).

We hypothesised that the spatial positioning of the positively charged quaternary ammonium group of $\mathrm{KMe} 3 / \mathrm{OrnMe} 3 / \mathrm{hKMe} 3$ inside the electron-rich aromatic cage of reader proteins determines the strength of cation $-\pi$ interactions and the overall binding affinity. The importance of cation $-\pi$ interactions in biomolecular recognition has been well established; ${ }^{12-15}$ they appear as a dominant driving force for the recognition of quaternary ammonium cations in several biologically relevant protein-ligand interactions. ${ }^{16-19}$

Fmoc-protected OrnMe3 and hKMe3 were synthesised from Fmoc-Orn-OH and Fmoc-hK(Boc)-OH, and subsequently incorporated into the position 4 of histone $\mathrm{H} 3$ peptide (sequence: ARTXQTARKS) (Fig. 1C, and Scheme S1 and Fig. S1-8 in ESI). Synthetic histone peptides $\mathrm{H} 3 \mathrm{~K} 4 \mathrm{Me} 3, \mathrm{H} 3 \mathrm{Orn} 4 \mathrm{Me}$, and $\mathrm{H} 3 \mathrm{hK} 4 \mathrm{Me} 3$ were then examined for binding with five human reader proteins, which belong to the family of PHD zinc fingers (KDM5A, TAF3 and BPTF) and tandem tudor domains (SGF29 and KDM4A), employing isothermal titration calorimetry (ITC) (Table 1 and Fig. S9, ESI).

Our ITC experiments revealed that H3Orn4Me3 bound to reader proteins with 4-19 fold lower affinity than $\mathrm{H} 3 \mathrm{~K} 4 \mathrm{Me} 3$ (Table 1). The decreased binding affinity of H3Orn4Me3 relative to $\mathrm{H} 3 \mathrm{~K} 4 \mathrm{Me} 3$ is a result of less favourable enthalpy of binding $\left(\Delta H^{\circ}\right)$, which we attribute to weaker cation $-\pi$ interactions. Measured values of the incremental enthalpy of binding $\left(\Delta \Delta H^{\circ}\right)$ are found to be between $2.8 \mathrm{kcal} \mathrm{mol}^{-1}$ (for the PHD3 domain of KDM5A) to $9.4 \mathrm{kcal} \mathrm{mol}^{-1}$ (for the TTD of KDM4A). Thermodynamic data, moreover, showed that the entropic component of binding ($\mathrm{T} \Delta S^{\circ}$ ) is more favourable for $\mathrm{H} 3 \mathrm{Orn} 4 \mathrm{Me} 3$ than for $\mathrm{H} 3 \mathrm{~K} 4 \mathrm{Me} 3 ;-\mathrm{T} \Delta \Delta S^{\circ}$ values are in the range of $-1.0 \mathrm{kcal} \mathrm{mol}^{-1}$ (for KDM5A) to $-8.6 \mathrm{kcal} \mathrm{mol}^{-1}$ (for KDM4A). ITC data, furthermore, demonstrated that the association between reader proteins and $\mathrm{H} 3 \mathrm{hK} 4 \mathrm{Me} 3$ is stronger than the association with H3Orn4Me3, and comparable to the binding of H3K4Me3 (Table 1). KDM5A and KDM4A recognise equally well the one-methylene longer hKMe3 and KMe3. Other three readers bind the H3hK4Me3 with slightly weaker (2-5 fold) affinity. With the exception of KDM4A, other four reader proteins exhibit less favourable enthalpy of binding (about $0.8-4.0 \mathrm{kcal}$ $\mathrm{mol}^{-1}$ ) for $\mathrm{H} 3 \mathrm{hK} 4 \mathrm{Me} 3$ when compared to $\mathrm{H} 3 \mathrm{~K} 4 \mathrm{Me} 3$; the magnitude and trend could be a result of 
overall strong, but somewhat weaker cation $-\pi$ interactions. Consequently, $\Delta S^{\circ}$ is, in general, more favourable for the reader-H3hK4Me3 associations. Taken together, our thermodynamic analyses highlight that reader proteins exhibit strong binding affinity for H3Orn4Me3 and H3hK4Me3. Binding of both KMe3 analogues typically leads to less favourable enthalpy of binding (when compared to reader-H3K4Me3), an observation that is compatible with weaker cation- $\pi$ interactions between the positively charged quaternary ammonium moiety and the electron-rich aromatic cage of reader proteins.

Having shown that H3Orn4Me3 and H3hK4Me3 efficiently bind to reader proteins, we then carried out molecular dynamics (MD) simulations on the same panel of five readers complexed with H3Orn4Me3 and $\mathrm{H} 3 \mathrm{hK} 4 \mathrm{Me} 3$. The KMe3 residue of $\mathrm{H} 3 \mathrm{~K} 4 \mathrm{Me} 3$ in crystal structures of reader proteins was manually replaced with OrnMe 3 and hKMe3 residues (PDB IDs, see ESI), solvated in a $10 \AA$ truncated octahedral box of TIP3P water, ${ }^{20}$ and neutralised explicitly with either sodium or chloride ions. AMBER $12^{21}$ was then used to simulate the systems for $10 \mathrm{~ns}$ each, as recently described (Fig. S10-S25, ESI). ${ }^{9}$ A simulation of 50 ns was performed for highly flexible reader KDM5A with OrnMe3 and hKMe3 to validate simulation time of 10 ns (Fig. S26S29 and Table S1-S2, ESI).

The binding poses of OrnMe 3 and hKMe 3 with respect to their aromatic cages are shown at times $0 \mathrm{~ns}, 5 \mathrm{~ns}$, and $10 \mathrm{~ns}$ as well as the distance vs time graphs of each ${ }^{\square} \mathrm{N}^{+}$to the centroid of the aromatic residue side chain (Fig. S10-25, ESI). The positioning of OrnMe3 and hKMe3 residues within the aromatic pocket is similar throughout the simulation relative to the size of the reader aromatic cage (Fig. S30). BPTF has a single W32 that acts as a $\pi$ donor (Fig. S30A). This allows more flexibility to prioritize the stabilizing cation- $\pi$ interaction with the modified trimethylated lysine residues. KDM5A is characterised with having an aromatic cage composed of two tryptophans. Interestingly, a slight preference for W28 over W18 is shown by both residues (Fig. 2 and S30B). Reader TAF3 also contains dual adjacent facing tryptophan residues, however, unlike KDM5A the side chains are positioned as a mirror images rather than symmetrically (Fig. S30C). This creates a much wider conical shape that extends up to $11.1 \AA$, explaining the large deviation seen in the time vs. distance plots (Fig. S20-S21). KDM4A and SGF29 have a Phe-TrpTyr and Tyr-Phe-Tyr aromatic cage, respectively, creating a basket shape (Fig. S30D-E). The KDM4A binding site appears to be less rigid than SGF29, where the latter is composed of equidistant substituted benzenes. This parallels the differences seen for SGF29 OrnMe3, which is observed to be short from Tyr238, but stable. Additionally, a similar pattern is shown by hKMe3 and SGF29 F264, where due to a longer chain it is stabilizing outside the centre of the aromatic cage.

The electrostatic energies ( $\left.\Delta E_{\text {ele }}\right)$ between $N^{\varepsilon}$ of hKMe3 and OrnMe3 with the $\pi$-system of aromatic cages were also analysed (Table S4, ESI). Compared with the natural H3K4Me3, all readers complexed with OrnMe3 and hKMe3 had slightly weaker $\Delta E_{\text {ele }}$ values. hKMe3 indicated

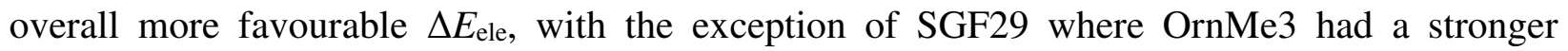
interaction with two of three aromatic cage residues.

Next, we quantum chemically analysed the energetics and bonding mechanism of TRP2 (a model for the two tryptophan residues of KDM5A; Fig. S31, see Section 6 in ESI) with OrnMe3 
and hKMe3, and compared to KMe3, using dispersion-corrected density functional theory at BLYP-D3BJ/TZ2P and COSMO for simulating aqueous solutions by means of ADF., 22 The new TRP2-OrnMe3 model complex presents a weaker bonding interaction than TRP2-KMe3 by 2.6 $\mathrm{kcal} \mathrm{mol}^{-1}: \Delta E(\mathrm{aq})=-7.6$ and $-10.2 \mathrm{kcal} \mathrm{mol}^{-1}$ for OrnMe3 and KMe3 complexes, respectively (Table 2). This finding agrees with our experimental observations. The reason must be found in the larger distance between $\mathrm{NMe}_{3}{ }^{+}$and TRP2 in the former. The bonding energy for the longer TRP2-hKMe3 is also slightly weaker $\left(-9.3 \mathrm{kcal} \mathrm{mol}^{-1}\right)$ than that of TRP2-KMe3, even though its $\mathrm{NMe}_{3}{ }^{+}$is closer to TRP2. However, an addition (hKMe3) or removal (OrnMe3) of one $\mathrm{CH}_{2}$ group from the chain causes a variation of the orientation of the system versus TRP2 (Fig. S32). In fact, whereas in $\mathrm{KMe} 3$ the $\mathrm{NMe}_{3}{ }^{+}$group is eclipsed with respect to the chain, in both OrnMe3 and hKMe3 the $\mathrm{NMe}_{3}{ }^{+}$group is staggered. This causes that, even though in hKMe3 the top methyl group of $\mathrm{NMe}_{3}{ }^{+}$is closer to TRP2, the rest of the chain is further away when compared to KMe3, thus justifying the weaker interaction discussed above. In fact, when one $\mathrm{CH}_{2}$ unit is either added or removed, having $\mathrm{C}_{\alpha}$ fixed, the rest of the side chain rotates with respect to KMe3. Such rotation is larger for OrnMe 3 than for hKMe3 due to the former being more inside into the cavity of TRP2. Note that in all three cases the equilibrium structure is the eclipsed one, as for KMe3 (Fig. S33). Our analyses show that side chains of KMe3, OrnMe3, and hKMe3 suffer almost negligible geometry deformation when they occupy the TRP2 cage: $\Delta E(\mathrm{aq})_{\text {strain }}=0.1,0.3$ and $0.8 \mathrm{kcal} \mathrm{mol}^{-1}$ for KMe3, OrnMe3, and hKMe 3 complexes, respectively. In the absence of aqueous solvation, the preference for KMe3 over OrnMe3 is preserved, while over hKMe3 is even increased $\left(\Delta E_{\text {int }}=-\right.$ 27.6, -25.1, and -25.2 $\mathrm{kcal} \mathrm{mol}^{-1}$ for KMe3, OrnMe3, and hKMe3 complexes, respectively).

Finally, we addressed the question why the TRP2 unit interacts more favourably with KMe3 than with either OrnMe3 or hKMe3, using quantitative Kohn-Sham molecular orbital (KSMO) and an associated energy decomposition analysis (EDA) of the referred intrinsic interaction energy $\Delta E_{\text {int }}$ (Table 2). First, the shorter OrnMe3 fragment leads to less Pauli repulsion (16.5 kcal $\mathrm{mol}^{-1}$ ) compared to the almost equal values for both KMe3 and hKMe3 (20.8 and $20.1 \mathrm{kcal} \mathrm{mol}^{-1}$, respectively), which agrees with the longer distance to TRP2 of the former. However, KMe3 presents more favourable attractive interactions (electrostatic, orbital and dispersion), that contribute to its stronger interaction (Table 2). These results are supported by the above discussed geometries of these complexes, i.e. closer proximity (better orientation) of the whole side chain to TRP2 is translated in more attractive electrostatic interaction $\left(\Delta V_{\text {elstat }}=-15.0\right.$ vs. -12.7 vs. -13.7 $\mathrm{kcal} \mathrm{mol}^{-1}$ for $\mathrm{KMe} 3$, OrnMe3, and hKMe3, respectively; values that perfectly support above discussed $\Delta E_{\text {ele }}$ for larger systems), also supported by the somewhat more positively charged $\mathrm{H}$ atoms of KMe3 (Fig. 3A). Furthermore, the better conformation for KMe3 also influences the more attractive $\Delta E_{\mathrm{oi}}$ term in TRP2-KMe3 $\left(-13.0 \mathrm{kcal} \mathrm{mol}^{-1}\right)$ than TRP2-OrnMe3 and TRP2hKMe3 (-12.0 and $-11.3 \mathrm{kcal} \mathrm{mol}^{-1}$, respectively), which means stronger, more stabilizing donoracceptor orbital interactions from TRP $2 \pi$ orbitals to acceptor $\sigma^{*} \mathrm{C}-\mathrm{H}$ type orbitals on the KMe3 side chain (Table 2, Fig. 3B). Charge transfer is 0.04 electrons to KMe3, 0.03 to OrnMe3, and 0.04 to hKme3. This preference for KMe 3 is also caused by the better overlap between TRP $2 \pi$ orbitals and the acceptor orbitals of KMe3. As can be seen in Table S5 (ESI), the overlap between HOMO of TRP2 and LUMO+1 of KMe3 (0.028) is larger than for either OrnMe3 (0.012) or hKMe3 (0.025). As a whole, the EDA proves a direct relationship between the binding energy and the geometry, i.e. KMe3 has the right length to optimally interact with TRP2, proven with the larger 
$\Delta E_{\text {Pauli. }}$ On the other hand, the shorter OrnMe3 reaches less deep into TRP2, thus causing weaker interactions (less $\Delta E_{\text {Pauli, }}$, but also weaker attractive $\Delta V_{\text {elstat }}$ and $\Delta E_{\mathrm{oi}}$ ), whereas longer hKMe3 exhibits a nonoptimal binding mode, and thus also causing all interaction terms to be slightly less favourable.

Overall, our thermodynamic data, molecular dynamics simulations, and quantum chemical analyses reveal that $N^{\varepsilon}$-methyllysine binding reader proteins interact with histones that possess trimethylornithine (relatively weaker complex) and trimethylhomolysine (strong complex), the simplest shorter and longer analogues of naturally existing $N^{\varepsilon}$-trimethyllysine. Binding of OrnMe3 and hKMe3 is typically associated with less favourable $\Delta H^{\circ}, \Delta E_{\text {ele, }}$ and $\Delta E(\mathrm{aq})$, thus implying that nonoptimal positioning of the quaternary ammonium cations inside the aromatic cages of reader proteins leads to energetically weaker, but still strong, cation $-\pi$ interactions. In contrast to functionally related histone lysine methyltransferases that are very sensitive to changes in the length of the side chain that undergoes the enzymatic methylation, ${ }^{23} N^{\varepsilon}$-methyllysine binding proteins exhibit a broader tolerance for the acceptance of shorter and longer $N^{\varepsilon}$-trimethyllysine analogues. The signature for binding of KMe3, OrnMe3, and hKMe3 by reader proteins, nonetheless, resembles recent findings that human trimethyllysine hydroxylase, the first enzyme of carnitine biosynthesis pathway, does efficiently catalyse C-3 hydroxylation of these three methylated compounds in the free form. ${ }^{24}$ Our study is important not only because it demonstrates the power of synergistic experimental and computational chemical approaches for examining the biomolecular recognition in epigenetics, but also because it may guide rational design of novel chemical probes of $N^{\varepsilon}$-methyllysine binding readers.

This work was supported by the ERC Starting Grant to J. M. (ChemEpigen-715691) and the Netherlands Organization for Scientific Research (NCI-TA 731.015.202). K. K. is supported by a World Bank Education Grant. J. P. thanks the Spanish MINECO (CTQ2016-77558-R).

\section{Conflicts of interest}

There are no conflicts to declare.

\section{References}

1 A. J. Bannister and T. Kouzarides, Cell Res., 2011, 21, 381-395.

2 J. C. Black, C. van Rechem and J. R. Whetstine, Mol. Cell, 2012, 48, 491-507.

3 C. Qian and M.-M. Zhou, Cell. Mol. Life Sci., 2006, 63, 2755-2763.

4 S. M. Kooistra and K. Helin, Nat. Rev. Mol. Cell Biol., 2012, 13, 297-311.

5 S. D. Taverna, H. Li, A. J. Ruthenburg, C. D. Allis and D. J. Patel, Nat. Struct. Mol. Biol., 2007, 14, 1025-1040. 
6 R. M. Hughes, K. R. Wiggins, S. Khorasanizadeh and M. L. Waters, Proc. Natl. Acad. Sci. USA, 2007, 104, 11184-11188.

7 J. J. A. G. Kamps, J. Huang, J. Poater, C. Xu, B. J. G. E. Pieters, A. Dong, J. Min, W. Sherman, T. Beuming, F. M. Bickelhaupt, H. Li and J. Mecinović, Nat. Commun., 2015, 6, 8911.

$8 \quad$ R. J. Sims and D. Reinberg, Genes Dev., 2006, 20, 2779-2786.

9 R. Belle, A. H. K. Al Temimi, K. Kumar, B. J. G. E. Pieters, A. Tumber, J. E. Dunford, C. Johansson, U. Oppermann, T. Brown, C. J. Schofield, R. J. Hopkinson, R. S. Paton, A. Kawamura and J. Mecinović, Chem. Commun., 2017, 53, 13264-13267.

10 D. Seeliger, S. Soeroes, R. Klingberg, D. Schwarzer, H. Grubmüller and W. Fischle, ACS Chem. Biol., 2012, 7, 150-154.

11 M. D. Simon, F. Chu, L. R. Racki, C. C. de la Cruz, A. L. Burlingame, B. Panning, G. J. Narlikar and K. M. Shokat, Cell, 2007, 128, 1003-1012.

12 K. D. Daze and F. Hof, Acc. Chem. Res., 2013, 46, 937-945.

13 D. A. Dougherty, Science, 1996, 271, 163-168.

14 D. A. Dougherty, Acc. Chem. Res., 2013, 46, 885-893.

15 J. C. Ma and D. A. Dougherty, Chem. Rev., 1997, 97, 1303-1324.

16 J. J. A. G. Kamps, A. Khan, H. Choi, R. K. Lesniak, J. Brem, A. M. Rydzik, M. A. McDonough, C. J. Schofield, T. D. W. Claridge and J. Mecinović, Chem. Eur. J., 2016, 22, 12701276.

17 L. M. Salonen, C. Bucher, D. W. Banner, W. Haap, J.-L. Mary, J. Benz, O. Kuster, P. Seiler, W. B. Schweizer and F. Diederich, Angew. Chem. Int. Ed., 2009, 48, 811-814.

18 G. N. Nagy, L. Marton, A. Contet, O. Ozohanics, L.-M. Ardelean, Á. Révész, K. Vékey, F. D. Irimie, H. Vial, R. Cerdan and B. G. Vértessy, Angew. Chem. Int. Ed., 2014, 53, 1347113476.

19 J. Sussman, M. Harel, F. Frolow, C. Oefner, A. Goldman, L. Toker and I. Silman, Science, $1991,253,872-879$.

20 W. L. Jorgensen, J. Chandrasekhar, J. D. Madura, R. W. Impey and M. L. Klein, J. Chem. Phys., 1983, 79, 926-935.

21 D. A. Case et al., AMBER 12, University of California, San Francisco, 2012.

22 G. te Velde, F. M. Bickelhaupt, E. J. Baerends, C. Fonseca Guerra, S. J. A. van Gisbergen, J. G. Snijders and T. Ziegler, J. Comput. Chem., 2001, 22, 931-967.

23 A. H. K. A. Temimi, Y. V. Reddy, P. B. White, H. Guo, P. Qian and J. Mecinović, Sci. Rep., 2017, 7, 16148. 
24 A. H. K. Al Temimi, B. J. G. E. Pieters, Y. V. Reddy, P. B. White and J. Mecinović, Chem. Commun., 2016, 52, 12849-12852. 


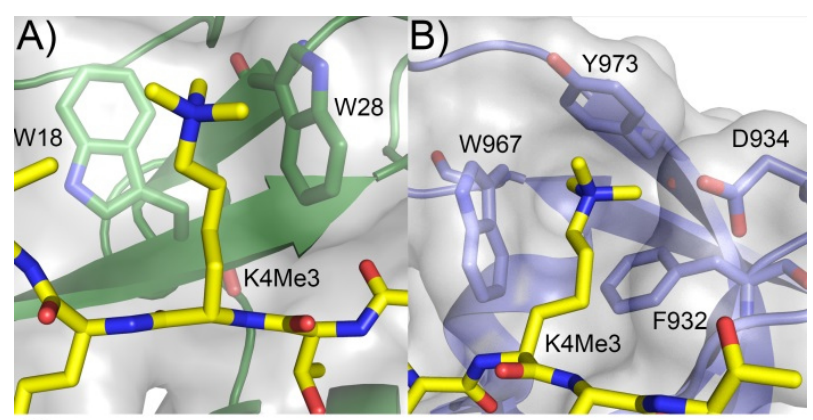

C)
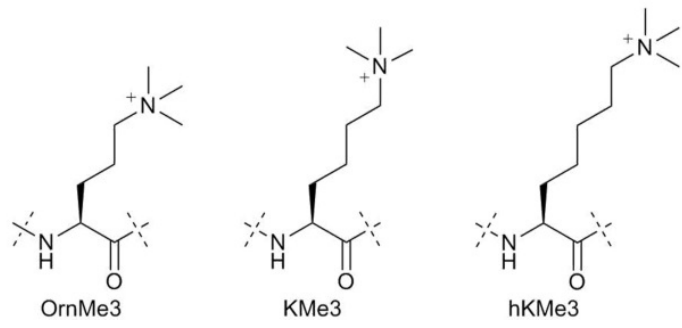

Fig. 1 Recognition of $N^{\varepsilon}$-trimethyllysine by reader proteins. A) View from a KDM5APHD3 (green) structure complexed with histone H3K4Me3 (yellow). B) View from a KDM4ATTD (blue) structure complexed with histone H3K4Me3 (yellow). C) Structures of $N^{\varepsilon}$-trimethyllysine, trimethylornithine and trimethylhomolysine. 

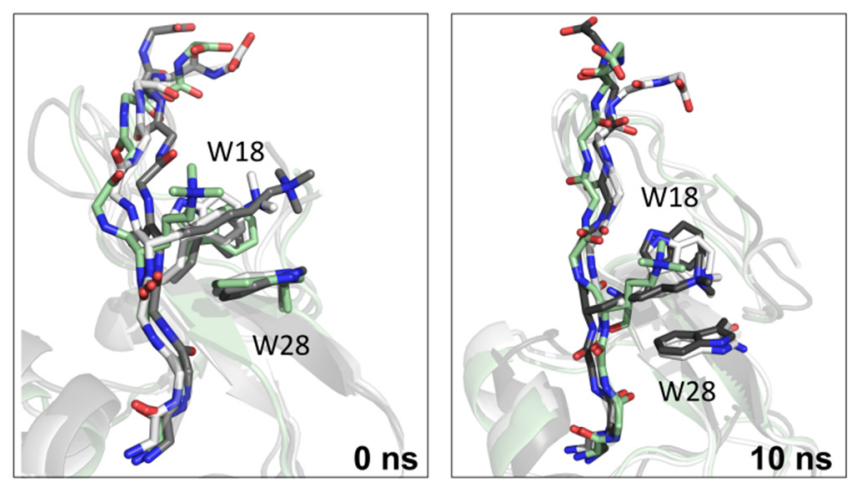

Fig. 2 MD simulations on H3Orn4Me3 (green), H3K4Me3 (white), and H3hK4Me3 (grey) with KDM5A at $0 \mathrm{~ns}$ and $10 \mathrm{~ns}$. 


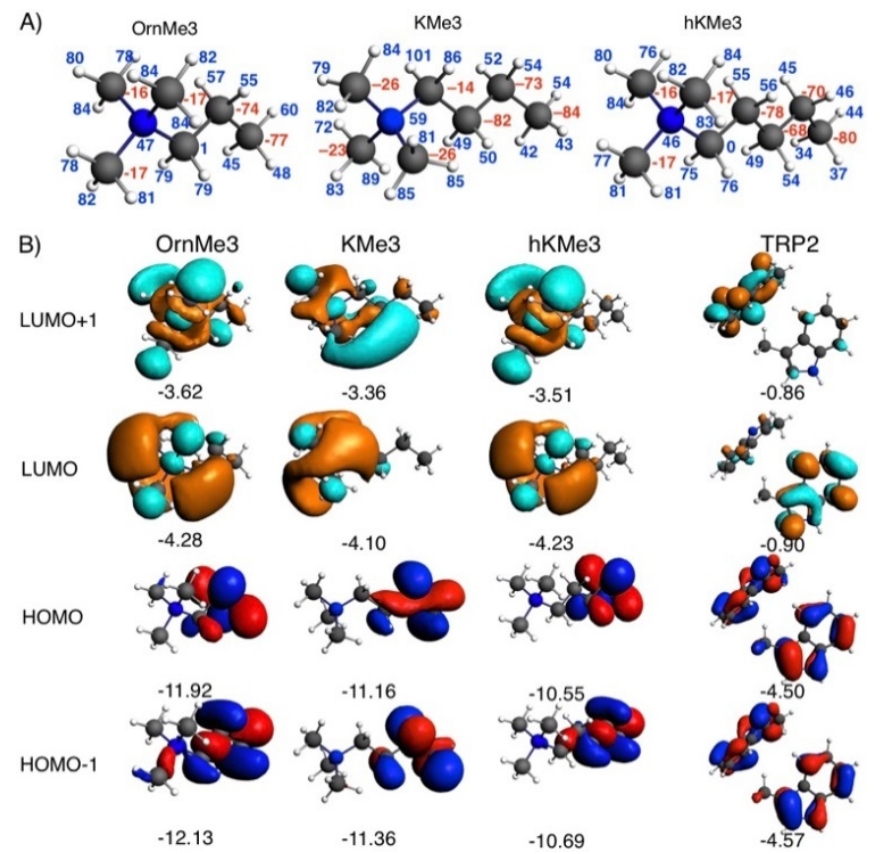

Fig. 3 Computational analysis of TRP2-OrnMe3, TRP2-KMe3, and TRP2-hKMe3 interactions. A) VDD atomic charges (in mili-a.u.) of OrnMe3, KMe3 and hKMe3 (red = negative, blue = positive). B) Frontier orbitals (with orbital energies in eV) of KMe3, OrnMe3, hKMe3, and TRP2, (isosurface drawn at 0.03). Computed at BLYP-D3BJ/TZ2P using X-ray structure for TRP2, and fully relaxed for the rest. 
Table 1 Thermodynamic parameters for binding of 10-mer histone peptides H3Orn4Me3, $\mathrm{H} 3 \mathrm{~K} 4 \mathrm{Me} 3$, and $\mathrm{H} 3 \mathrm{hK} 4 \mathrm{Me} 3$ to five reader proteins.

\begin{tabular}{|c|c|c|c|c|c|}
\hline \multicolumn{2}{|c|}{ ARTXQTTARKS } & \multirow{2}{*}{$\begin{array}{l}K_{\mathrm{d}} \\
(\mu \mathrm{M})\end{array}$} & \multirow{2}{*}{$\begin{array}{l}\Delta G^{\circ} \\
\left(\mathrm{kcal} \mathrm{mol}^{-1}\right)\end{array}$} & \multirow{2}{*}{$\begin{array}{l}\Delta H^{\circ} \\
\left(\mathrm{kcal} \mathrm{mol}^{-1}\right)\end{array}$} & \multirow{2}{*}{$\begin{array}{l}-\mathrm{T} \Delta S^{\circ} \\
\left(\mathrm{kcal} \mathrm{mol}{ }^{-1}\right)\end{array}$} \\
\hline & $X=$ & & & & \\
\hline \multirow[t]{3}{*}{ KDM5A } & OrnMe3 & 10 & $-6.8 \pm 0.1$ & $-7.8 \pm 0.1$ & $1.0 \pm 0.1$ \\
\hline & KMe3 & 0.52 & $-8.6 \pm 0.1$ & $-10.6 \pm 0.1$ & $2.0 \pm 0.1$ \\
\hline & hKMe3 & 0.36 & $-8.8 \pm 0.1$ & $-9.8 \pm 0.1$ & $1.0 \pm 0.1$ \\
\hline \multirow[t]{3}{*}{ TAF3 } & OrnMe3 & 1.0 & $-8.2 \pm 0.1$ & $-7.3 \pm 0.1$ & $-0.86 \pm 0.1$ \\
\hline & KMe3 & 0.082 & $-9.7 \pm 0.1$ & $-10.1 \pm 0.1$ & $0.37 \pm 0.1$ \\
\hline & hKMe3 & 0.41 & $-8.7 \pm 0.1$ & $-7.5 \pm 0.1$ & $-1.2 \pm 0.1$ \\
\hline \multirow[t]{3}{*}{ BPTF } & OrnMe3 & 18 & $-6.5 \pm 0.1$ & $-5.7 \pm 0.1$ & $-0.8 \pm 0.1$ \\
\hline & KMe3 & 1.4 & $-8.0 \pm 0.1$ & $-12.4 \pm 0.1$ & $4.4 \pm 0.1$ \\
\hline & hKMe3 & 3.1 & $-7.5 \pm 0.1$ & $-8.0 \pm 0.1$ & $0.5 \pm 0.1$ \\
\hline \multirow[t]{3}{*}{ SGF29 } & OrnMe3 & 19 & $-6.4 \pm 0.1$ & $-4.1 \pm 0.1$ & $-2.3 \pm 0.1$ \\
\hline & KMe3 & 3.1 & $-7.5 \pm 0.1$ & $-8.1 \pm 0.1$ & $0.64 \pm 0.1$ \\
\hline & hKMe3 & 7.3 & $-7.0 \pm 0.1$ & $-4.0 \pm 0.1$ & $-3.0 \pm 0.1$ \\
\hline \multirow[t]{3}{*}{ KDM4A } & OrnMe3 & 15 & $-6.6 \pm 0.2$ & $-3.2 \pm 0.2$ & $-3.4 \pm 0.2$ \\
\hline & KMe3 & 4.0 & $-7.4 \pm 0.1$ & $-12.6 \pm 0.1$ & $5.2 \pm 0.1$ \\
\hline & hKMe3 & 5.7 & $-7.2 \pm 0.1$ & $-12.9 \pm 0.1$ & $5.7 \pm 0.1$ \\
\hline
\end{tabular}


Table 2 Quantum-chemical bonding analysis (energies in $\mathrm{kcal} \mathrm{mol}^{-1}$, distances in $\AA$ ) in TRP2OrnMe3, TRP2-KMe3, and TRP2-hKMe3 systems in aqueous solution. ${ }^{[a]}$

\begin{tabular}{lccc}
\hline & TRP2-OrnMe3 $^{[\mathrm{b}]}$ & TRP2-KMe3 $^{[\mathrm{cc}]}$ & TRP2-hKMe3 $^{[\mathrm{b}]}$ \\
\hline$\Delta E$ (aq) & -7.6 & -10.2 & -9.3 \\
$\Delta E(\mathrm{aq})_{\text {strain }}$ & 0.3 & 0.1 & 0.8 \\
$\Delta E$ (aq) $)_{\text {int }}$ & -7.9 & -10.3 & -10.1 \\
$\Delta E{\text { (desolv })_{\text {int }}}$ & 17.2 & 17.3 & 15.1 \\
$\Delta E_{\text {int }}$ & -25.1 & -27.6 & -25.2 \\
$\Delta E_{\text {Pauli }}$ & 16.5 & 20.8 & 20.1 \\
$\Delta V_{\text {elstat }}$ & -12.7 & -15.0 & -13.7 \\
$\Delta E_{\text {oi }}$ & -12.0 & -13.0 & -11.3 \\
$\Delta E_{\text {disp }}$ & -16.8 & -20.4 & -20.2 \\
$\mathrm{~d}\left(\mathrm{H}_{\text {Me-CTRP-6MR }}\right)$ & 3.16 & 2.88 & 2.67 \\
$\mathrm{~d}\left(\mathrm{H}_{\text {Me-C }}-C_{\text {TRP-5MR }}\right)$ & 3.33 & 2.78 & 2.66 \\
\hline
\end{tabular}

al Computed at BLYP-D3BJ/TZ2P with COSMO to simulate aqueous solution. Structural rigidity imposed by the protein backbone is simulated through constrained geometry optimizations. See also Eqs. 1-3 in ESI. [b] TRP2 frozen, $\alpha$-methyl carbon fixed to position in TRP2-KMe3 optimization. ${ }^{[c]}$ TRP2 frozen, KMe3 entirely free. 